\title{
Electroweak symmetry breaking and cold dark matter from strongly interacting hidden sector
}

\section{Pyungwon Ko*i}

School of Physics, KIAS, Seoul 130-722, Korea

E-mail: pko@kias.re.kr

\begin{abstract}
We consider a hidden sector with new vectorlike confining gauge theories like QCD. Then a scale $\Lambda_{H}$ would be generated in the hidden sector by dimensional transmutation, and chiral symmetry breaking occurs in the hidden sector. Then this scale $\Lambda_{H}$ can play a role of the SM Higgs mass parameter, triggering electroweak symmetry breaking (EWSB). Thus all the mass scales of the SM sector can arise from the hidden sector. Furthermore the lightest hadrons in the hidden sector is stable by the flavor conservation of the hidden sector strong interaction, and could be the cold dark matter (CDM). We study collider phenomenology, and relic density and direct detection rates of the CDM of this model.
\end{abstract}

35th International Conference of High Energy Physics

July 22-28, 2010

Paris, France

\footnotetext{
${ }^{*}$ Speaker.

$\dagger$ This work was supported in part by Korea Neutrino Research Center (KNRC) of Seoul National University through National Research Foundation of Korea Grant.
} 


\section{Introduction}

Revealing the origin of the electroweak symmtry breaking (EWSB) is the most pressing question in particle physics in the era of CERN Large Hadron Collider (LHC). Another important problem in particle astrophysics and cosmology is to identify the nature of cold dark matter (CDM). Also there is a more speculative issue about the existence of a new hidden sector, which is generic in supersymmetric (SUSY) model buildings or superstring theories. In this talk, I would like to consider three seemingly unrelated questions:

- Can all the masses arise (mostly) from quantum mechanics, as in massless QCD ?

- What is the nature of CDM ? Is it possible to have all the global symmetry as accidental symmetries, as in the standard model (SM)?

- What would be the phenomenological consequences, if there is a hidden sector ?

I will present models with a hidden sector where these seemingly unrelated questions are in fact closely connected with each other. More details and complete list of references can be found in Ref.s [1,2].

Let me remind you that there is a good old example, namely quantum chromodynamics(QCD), where we can learn many lessons related with the issues listed above. QCD has many nice features: renormalizability, asymptotic freedom, confinement and chiral symmetry breaking, dynamical generation of hadron masses, natural hierarchy between the Planck scale and the QCD scale QCD $_{\text {. In }}$ addition pions are stable if electroweak interactions are switched off. It would be nice if we could have a model for EWSB in the same manner as the dimensional transmutation in QCD, and CDM is stable as pions are stable under strong interaction.

The basic features of our models are the following. We assume a vectorlike confining gauge theory such as QCD in the hidden sector. Then dimensional transmutation will occur in the hidden sector, and this scale is transmitted to the SM by a messenger, and triggers EWSB. And the lightest mesons in the hidden sector becomes a CDM.

\section{Model I}

Let us assume that there is a new strong interaction that is described by $S U\left(N_{h, C}\right)$ guage theory with vectorlike quarks $\mathscr{Q}_{i}$ and $\overline{\mathscr{Q}}_{i}$ with $N_{h, f}$ flavors, such as QCD with the confinement scale $\Lambda_{h}$. This scale is presumed to be higher than the electroweak scale by at least an order of magnitude.

$$
\mathscr{L}_{\text {hidden }}=-\frac{1}{4} \mathscr{G}_{\mu v} \mathscr{G}^{\mu v}+\sum_{k=1}^{N_{H F}} \overline{\mathscr{Q}}_{k}\left(i \mathscr{D} \cdot \gamma-M_{k}\right) \mathscr{Q}_{k}
$$

Then this new strong interaction will trigger chiral symmetry breaking due to nonzero $\langle\overline{\mathscr{Q}}\rangle \equiv$ $\Lambda_{H, \chi}^{3}$. For illustration, we assume that there is an approximate $S U(2)_{L} \times S U(2)_{R}$ global symmetry in the hidden sector that breaks down to $S U(2)_{V}$ spontaneously. Then at low energy scale, massless Nambu-Goldstone bosons will appear, which we call hidden sector pions $\pi_{h}$ 's. Also there would be a scalar resonance like the ordinary $\sigma$, and we call it $\sigma_{h}$, and $\vec{\pi}_{h}$ and $\sigma_{h}$ will form $S U(2)_{L} \times S U(2)_{R}$ 

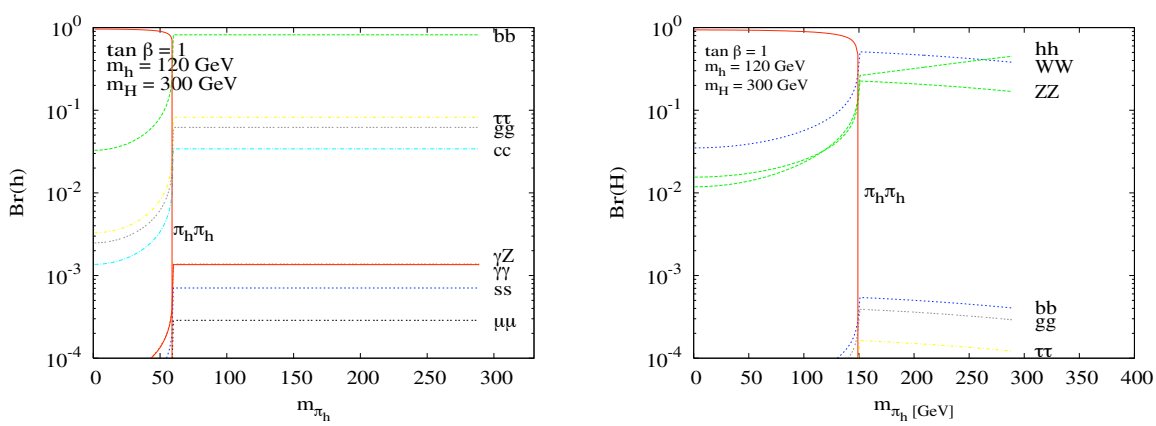

Figure 1: Branching ratios of (a) $h$ and (b) $H$ as functions of $m_{\pi_{h}}$ for $\tan \beta=1, m_{h}=120 \mathrm{GeV}$ and $m_{H}=300 \mathrm{GeV}$.

bidoublet (denoted as $\mathrm{H}_{2}$ ) and the low energy effective theory will be the same as the GelmannLevy's linear $\sigma$ model, except that the mesons $\left(\pi_{h}\right.$ 's) are all in the hidden sector, so that they are all SM singlets.

The potential for the SM Higgs and the hidden sector $H_{2}$ is given by

$$
V\left(H_{1}, H_{2}\right)=-\mu_{1}^{2}\left(H_{1}^{\dagger} H_{1}\right)+\frac{\lambda_{1}}{2}\left(H_{1}^{\dagger} H_{1}\right)^{2}-\mu_{2}^{2}\left(H_{2}^{\dagger} H_{2}\right)+\frac{\lambda_{2}}{2}\left(H_{2}^{\dagger} H_{2}\right)^{2}+\lambda_{3}\left(H_{1}^{\dagger} H_{1}\right)\left(H_{2}^{\dagger} H_{2}\right)+\frac{a v_{2}^{3}}{2} \sigma_{h}
$$

This looks like the potential in the 2-Higgs doublet model, but there are important differences. First, $H_{2}$ is a SM singlet, not a SM doublet. $W$ and $Z^{0}$ get masses entirely from $H_{1}$ VEV. And the $a$ term is new in our model, and necessary to generate the mass for the hidden sector pion. Note that the $\lambda_{3}$ term connects the SM and the hidden sector, and originates from nonrenormalizable interactions between two sectors, or by some messengers.

It is straightforward to analyze phenomenology from this scalar potential. The generic features of our models can be summarized as follows. The origin of the EWSB, namely the negative Higgs mass ${ }^{2}$ parameter could be the chiral symmetry breaking in the strongly interacting hidden sector. The electroweak precision test does not put strong constraints unlike in the ordinary technicolor models, since $\mathrm{H}_{2}$ does not contribute to the $W$ and $Z^{0}$ masses at tree level. And no Higgs-mediated FCNC problem since $H_{2}$ does not couple to the SM fermions. There are more than one neutral Higgs-like scalar bosons, and they can decay into the $\pi_{h}$ with a large invisible branching ratio. This makes relatively difficult to produce and discover these Higgs-like neutral scalars at colliders. See Fig. 1 (a) and (b). Finally, the hidden sector pion $\left(\pi_{h}\right)$ is stable due to the flavor conservation of hidden sector strong interaction, and could be a good CDM candidate. Direct detection rate of the $\pi_{h}$ is promisingly within the sensitivity of the current/future DM detection experiments.

\section{Model II with classical scale invariance}

The Model I has a few drawbacks, since the hidden sector quark masses $M_{k}$ 's are given by hand, and the Model I is not renormalizable. These can be cured by introducing a real singlet scalar $S$ and making the following replacement, $M_{k} \rightarrow \lambda_{k} S$ in Eq. (1). Then $\mathscr{L}_{\text {hidden }}$ has classical scale symmetry. With a real singlet $S$, the SM lagrangian is implemented into

$$
\mathscr{L}_{\text {SM }}=\mathscr{L}_{\text {kin }}+\mathscr{L}_{\text {Yukawa }}-\frac{\lambda_{H}}{4}\left(H^{\dagger} H\right)^{2}-\frac{\lambda_{S H}}{2} S^{2} H^{\dagger} H-\frac{\lambda_{S}}{4} S^{4}
$$



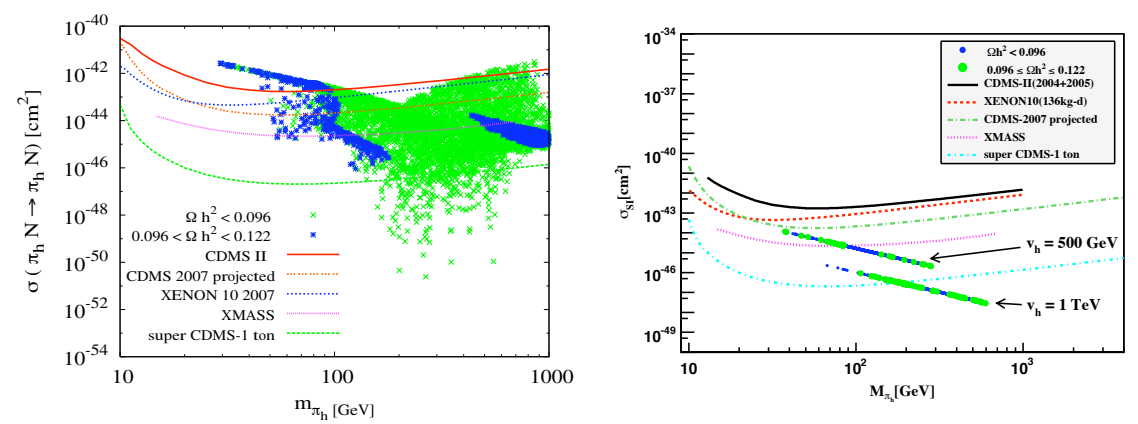

Figure 2: $\sigma_{S I}\left(\pi_{h} p \rightarrow \pi_{h} p\right)$ as functions of $m_{\pi_{h}}$ for (a) $\tan \beta=1$ in Model I, and (b) Model II.

assuming classical scale symmetry. Since there are no mass parameters in this lagrangian, this is a suitable starting point to investigate if it is possible to have all the masses from quantum mechanical effects. Note that the real singlet scalar $S$ plays the role of messenger connecting the SM Higgs sector and the hidden sector quarks.

Dimensional transmutation in the hidden sector will generate the hidden QCD scale and chiral symmetry breaking with developing nonzero $\left\langle\overline{\mathscr{Q}}_{k} \mathscr{Q}_{k}\right\rangle$. Then the $\lambda_{k} S$ term generate the linear potential for the real singlet $S$, leading to nonzero $\langle S\rangle$. This in turn generates the hidden sector current quark masses through $\lambda_{k}$ terms as well as the EWSB through $\lambda_{S H}$ term. The $\pi_{h}$ will get nonzero masses, and becomes a good CDM candidate. Due to the presence of the messenger $S$, the CDM pair annihilation into the SM particles occurs more efficiently in Model II than in Model I, and it is easy to accommodate the WMAP data on $\Omega_{\mathrm{CDM}} h^{2}$. Direct detection rates are in the interesting ranges (see Fig. 2 (b)). All the qualitative features of this model is similar to the Model I. See Ref. [2] for more details.

\section{Conclusions}

In this talk, I presented models where the origin of EWSB and CDM lie in the hidden sector technicolor interaction. In the Model II, all the masses including the CDM mass arise quantum mechanically from dimensional transmutation in the hidden sector. One can enjoy many variations of these models by considering different gauge groups and matter fields in the hidden sector. If we include the radiative corrections to the scalar potential, the details could change, but the qualitative features described in this talk would remain untouched.

\section{Acknowledgments}

I am grateful to Taeil Hur, D.W. Jung and J.Y. Lee for collaborations.

\section{References}

[1] T. Hur, D. W. Jung, P. Ko and J. Y. Lee, arXiv:0709.1218 [hep-ph].

[2] S. Baek, T. Hur and P. Ko, In preparation. 